\title{
Expedited Yield Optimization of Narrow- and Multi-Band Antennas Using Performance- Driven Surrogates
}

\author{
Anna Pietrenko-Dabrowska ${ }^{1}$, Senior Member, Slawomir Koziel ${ }^{1,2}$, Senior Member, IEEE, \\ IEEE, Muath Al-Hasan', Senior Member, IEEE \\ ${ }^{1}$ Faculty of Electronics, Telecommunications and Informatics, Gdansk University of Technology, Narutowicza 11/12, 80-233 Gdansk Poland \\ ${ }^{2}$ Engineering Optimization \& Modeling Center, Department of Engineering, Reykjavik University, Menntavegur 1, 102 Reykjavik, Iceland \\ ${ }^{3}$ Networks and Communication Engineering Department, Al Ain University, Abu Dhabi, United Arab Emirates \\ Corresponding author: Anna Pietrenko-Dabrowska (e-mail: anna.dabrowska@pg.edu.pl).
}

The authors would like to thank Dassault Systemes, France, for making CST Microwave Studio available. This work is partially supported by the Icelandic Centre for Research (RANNIS) Grant 206606051, by National Science Centre of Poland Grant 2017/27/B/ST7/00563, and by the Abu-Dhabi Department of Education and Knowledge (ADEK) Award for Research Excellence 2019 under Grant AARE19-245.

\begin{abstract}
Uncertainty quantification is an important aspect of engineering design, also pertaining to the development and performance evaluation of antenna systems. Manufacturing tolerances as well as other types of uncertainties, related to material parameters (e.g., substrate permittivity) or operating conditions (e.g., bending) may affect the antenna characteristics. In the case of narrow- or multi-band antennas, this usually leads to frequency shifts of the operating bands. Quantifying these effects is imperative to adequately assess the design quality, either in terms of the statistical moments of the performance parameters or the yield. Reducing the antenna sensitivity to parameter deviations is even more essential when increasing the probability of the system satisfying the prescribed requirements is of concern. The prerequisite of such procedures is statistical analysis, normally carried out at the level of full-wave electromagnetic (EM) analysis. While necessary to ensure reliability, it entails considerable computational expenses, often prohibitive. Following the recently fostered concept of constrained modeling, this paper proposes a simple technique for rapid surrogate-assisted yield optimization of narrow- and multi-band antennas. The keystone of the approach is an appropriate definition of the optimization domain. This is realized by considering a few pre-optimized designs that represent the directions of the major changes of the antenna resonant frequencies and operating bands. Due to a small volume of such a domain, an accurate replacement model can be established therein using a small number of training samples, and employed to improve the antenna yield. Verification results obtained for a ring-slot antenna, a dual-band and a triple-band uniplanar dipoles indicate that the optimization process can be accomplished at low cost of a few dozen of EM simulations: 62, 74 and 132 EM simulations, respectively. Result reliability is validated through comparisons with EM-based Monte Carlo simulations.
\end{abstract}

INDEX TERMS Uncertainty quantification; tolerance-aware design; yield optimization; multi-band antennas; performance-driven modeling.

\section{INTRODUCTION}

The vast majority of antenna design procedures aim at finding the nominal designs, i.e., obtained under the assumption that the fabricated prototype retains the values of geometry and/or material parameters equal to those rendered in the course of antenna development (e.g., through parametric optimization) [1]-[5]. In practice, uncertainties of various types may affect the system operation in an undesirable manner. Their appropriate quantification may therefore be crucial to verify whether the system is likely to satisfy the prescribed performance specifications. There are generally two types of uncertainties pertaining to antenna structures. The most common ones are deviations of antenna dimensions as well as the material parameters (e.g., substrate permittivity) from their nominal values. These are the inherent (or aleatory) uncertainties, most often related to manufacturing tolerances 
[6] and typically characterized by probability distributions. Due to their stochastic nature, quantification requires performing statistical analysis [7]-[9]. Reducing the effects of tolerances normally entails stochastic design that aims at improving statistical performance measures, e.g., the yield [10], [11]. The second type of uncertainties are systematic (or epistemic) ones, related to the lack of knowledge of the operating conditions (temperature, radius of bending of a wearable antenna, etc.). These may often be handled by ensuring that the acceptable system performance is secured for the specified ranges of the conditions.

Statistical analysis of antenna structures is a challenging endeavor mostly due to the necessity of using CPU-intensive full-wave electromagnetic (EM) analysis for reliable evaluation of performance parameters. Conventional statistical analysis routines, particularly Monte Carlo simulation [12], [13], involving massive EM analyses, entail considerable computational expenses, which typically turn prohibitive or at least make the analysis impractical. There have been many attempts to alleviate these difficulties in the literature. A simple approach is a worst-case analysis [14], [15], which can be realized without incurring excessive costs, yet, it more often than not provides overly pessimistic estimates. Nowadays, the most popular methods involve fast surrogate models such as response surface approximation [16], artificial neural networks [17], and, more and more popular, polynomial chaos expansion (PCE) [18]-[20]. The attractiveness of the latter originates from its convenience: PCE models allow for calculating the statistical moments of the system output directly from the expansion coefficients with no need for Monte Carlo analysis. One of the practical issues related to the use of surrogates is a potentially high cost of setting up the model, especially for higher-dimensional parameter spaces. Some of the recent approaches are arguably more economical in that sense, e.g., PC kriging [10], where low-order polynomial traditionally employed as a trend function is replaced by the PCE surrogate. Other possibilities include reduction of the problem dimensionality (e.g., using principal component analysis [21]), incorporating variablefidelity simulations by means of space mapping [22], or cokriging [23], as well as combinations of various approaches such as surrogate modeling and model order reduction [24].

Mitigating the effects of uncertainties on the antenna operation is an important design consideration. In practice, it requires the adjustment of geometry parameters so as to minimize the sensitivity of the figures of interest (center frequencies, bandwidths, axial ratio, etc.) to, e.g., manufacturing tolerances, or to maximize the probability of satisfying prescribed performance requirements. The latter is typically referred to as robust design, tolerance-aware design, yield-driven design, or design centering [25]-[30], depending on a particular explicit merit function (e.g., yield) being processed. Computational-wise, robust design is an expensive process because it normally involves multiple statistical analyzes encapsulated in an optimization loop [18]. Clearly, direct EM-based stochastic optimization is normally prohibitively expensive. In practice, the methods of choice involve surrogate models [6]-[31]. As mentioned in the previous paragraph, popular techniques include response surface approximations [14], space mapping [8], [22], [32] neural networks [8], and polynomial chaos expansion (PCE) [18]-[20], [33]-[34] . As yield-driven optimization may need to handle considerable ranges of the antenna parameters, a construction of reliable surrogates may become problematic, especially for higher-dimensional spaces. One of the workarounds is sequential approximate optimization (SAO) [35], where the surrogate is constructed locally with the domain relocated between iterations along the optimization path. Another option is the employment of the response feature approach [36], in which direct handling of original system characteristics (usually, S-parameters versus frequency) is replaced by constructing the surrogate at the level of suitably defined characteristic points. Reformulating the design task this way leads to a less nonlinear functional landscape, resulting in easier modeling that requires significantly smaller training data sets [37]-[39].

This paper proposes a novel and low-cost procedure for yield optimization of multi-band antennas. Our methodology employs the overall concept of performance-driven modeling [40]-[43] to construct a fast surrogate in the region corresponding to maximum changes of the antenna responses in the vicinity of the nominal design. By appropriate constraining of the model domain, the surrogate can be rendered at a very low cost of a few dozen of EM antenna analyzes while being valid over a sufficiently large parameter ranges to permit efficient optimization of the antenna yield. The presented framework is comprehensively validated using three test cases: a ring-slot antenna, a dual-band dipole antenna, and a triple-band dipole antenna. The performance of our algorithm is favorably compared to two surrogate-assisted approaches which involve: (i) a one-shot kriging surrogate constructed over a larger vicinity of the nominal design, and (ii) sequential approximate optimization with local kriging models set up along the optimization path.

The primary novelties and technical contributions of the paper, beyond what was proposed in the literature so far, include: (i) introduction of the concept of a surrogate domain confinement into the yield optimization process, (ii) a definition of a constrained domain based on the directions corresponding to the major variations of the antenna characteristics, (iii) demonstration of a considerable computational cost reduction of establishing an accurate replacement model for yield-optimization purposes within the constrained domain to as low as few dozen of EM simulations, (iv) demonstration of efficacy of the yield optimization procedure involving performance-driven surrogates, especially, a possibility of combining the advantages of oneshot and iterative (sequential approximation optimization) based techniques. Performing yield optimization of narrowand multi-band antennas at such a low cost and, at the same 
time, in a one-shot manner without neither design relocation nor surrogate re-building, has not been reported in the literature thus far.

\section{FAST YIELD OPTIMIZATION USING CONSTRAINED SURROGATES}

This section formulates the yield optimization problem as well as outlines the proposed algorithm for rapid tolerance-aware antenna design. The keystone of our method is a fast surrogate model constructed in a carefully defined constrained domain. The model exhibits good predictive power despite being based on small training data set. Furthermore, it is sufficiently flexible (in terms of the parameter space coverage) to be employed for reliable yield improvement.

\section{A. PROBLEM STATEMENT}

The problem of statistical analysis and tolerance-aware design can be formulated in various ways, depending on what type of statistical figures of merit are of interest for the designer. In this work, the focus is on multi-band antennas. We assume the minimax specifications for the input characteristics, specifically, given the target operating frequencies $f_{0 k}, k=1, \ldots, N$, and the target fractional bandwidth $B$. The antenna at the design $\boldsymbol{x}$ ( $\boldsymbol{x}$ stands for a vector of adjustable parameters) is said to satisfy the requirements if the following condition holds:

$$
\left\{f \in \bigcup_{k=1}^{N}\left[\frac{(1-B) f_{0 k}}{2}, \frac{(1+B) f_{0 k}}{2}\right]:\left|S_{11}(\boldsymbol{x}, f)\right|\right\} \leq S_{\max }
$$

where $f$ is the frequency, whereas $S_{\max }$ is typically $-10 \mathrm{~dB}$. The condition (1) means that the antenna matching is no worse than $S_{\max }$ within all target fractional bandwidths.

Let $\boldsymbol{x}^{(0)}$ be the nominal design and $d \boldsymbol{x}$ stand for a vector of deviations, e.g., manufacturing tolerances; $d \boldsymbol{x}$ is described by an assumed probability distribution, e.g., joint Gaussian with zero mean and variance $\sigma$, or uniform with maximum deviation $d_{\max }$. Generalization for other probability distribution (e.g., normal distributions described by a specific covariance matrix) is possible as well.

We define the function $H(\boldsymbol{x})$ which takes two values: 1 if the condition (1) is satisfied, and 0 otherwise. The estimated yield can be found by running Monte Carlo analysis as

$$
Y(\boldsymbol{x})=p^{-1} \sum_{k=1}^{p} H\left(\boldsymbol{x}^{(k)}\right)
$$

where $\boldsymbol{x}^{(k)}=\boldsymbol{x}+d \boldsymbol{x}^{(k)}, k=1, \ldots, p$, are random observables with $d \boldsymbol{x}^{(k)}$ being the random deviations as described above. The yield at the nominal design is then $Y\left(\boldsymbol{x}^{(0)}\right)$, and yield optimization problem is formulated as

$$
\boldsymbol{x}^{*}=\arg \min _{\boldsymbol{x}}\{-Y(\boldsymbol{x})\}
$$

Typically, the initial design for (3) is the nominal design $\boldsymbol{x}^{(0)}$, which may be obtained by solving a standard minimax problem of the form

$$
\begin{aligned}
& \boldsymbol{x}^{(0)}=\arg \min _{\boldsymbol{x}} \\
& \left\{\max \left\{f \in \bigcup_{k=1}^{N}\left[\frac{(1-B) f_{0 k}}{2}, \frac{(1+B) f_{0 k}}{2}\right]:\left|S_{11}(\boldsymbol{x}, f)\right|\right\}\right\}
\end{aligned}
$$

The solution to (4) determines the design that exhibits the best possible antenna matching within the operating bands of interest.

\section{B. REFERENCE ALGORITHMS}

As mentioned in the introduction, perhaps the most efficient option for EM-driven statistical analysis and robust design of antennas is the use of fast surrogate models. For the purpose of benchmarking the proposed procedure (Section II.C), the following two surrogate-assisted algorithms are considered:

Algorithm 1: Construct a kriging surrogate in a vicinity of the nominal design of the size $\boldsymbol{d}=\left[d_{1} \ldots d_{n}\right]^{T}$ (i.e., the interval $\left.\left[\boldsymbol{x}^{(0)}-\boldsymbol{d}, \boldsymbol{x}^{(0)}+\boldsymbol{d}\right]\right)$ and solve the yield maximization problem (3) therein. In order to create a sufficient room for yield improvement, the size of the mentioned vicinity should be sufficiently large. Here, we assume that $d_{k}=10 d_{\max }, k=1$, $\ldots, n$, where $d_{\max }$ is the maximum deviation in the case of uniform distribution, or $3 \sigma$ for Gaussian distribution of variance $\sigma$. This algorithm is very simple to implement but the cost of constructing an accurate surrogate model may be considerable due to the domain size.

Algorithm 2: Sequential approximate optimization (SAO). Replace (3) by an iterative process

$$
\boldsymbol{x}^{(i+1)}=\arg \min _{\boldsymbol{x}}\left\{-Y_{s}^{(i)}(\boldsymbol{x})\right\}
$$

where $\boldsymbol{x}^{(i)}, i=0,1, \ldots$, are the approximations of $\boldsymbol{x}^{*}$, whereas $Y_{s}^{(i)}$ is the yield estimated using the $i$ th surrogate model constructed in the vicinity $\boldsymbol{x}^{(i)}-\boldsymbol{d}_{l} \leq \boldsymbol{x} \leq \boldsymbol{x}^{(i)}-\boldsymbol{d}_{l}$ of the current design $\boldsymbol{x}^{(i)}=\left[x_{1}{ }^{(i)} \ldots x_{n}{ }^{(i)}\right]^{T}$. In this case, the model domain is smaller, say, $3 d_{\max }$, and it is being relocated between the algorithm iterations. Solving the problem (5) is subjected to constraints $x_{k}^{(i)}-d_{l . k}+d_{\max } \leq x_{k} \leq x_{k}^{(i)}+d_{l . k}-d_{\max }, k=1, \ldots$, $n$, to ensure that the point $\boldsymbol{x}$ is at least at the distance $d_{\max }$ from the surrogate model domain boundary (in all directions). In this case, the computational cost of constructing a reliable surrogate is much lower than for Algorithm 1, but a few iterations are necessary to conclude the optimization process. The algorithm is terminated if the current iteration does not improve the yield, i.e., if $Y_{s}^{(i+1)}\left(\boldsymbol{x}^{(i+1)}\right) \leq Y_{s}^{(i)}\left(\boldsymbol{x}^{(i)}\right)$. 


\section{YIELD OPTIMIZATION USING PERFORMANCE-DRIVEN SURROGATES}

The reference algorithms outlined in Section II.B represent the two extreme strategies for yield optimization, i.e., single surrogate model constructed over a larger portion of the parameter space versus an iterative process with the surrogates constructed over smaller regions relocated along the optimization path. The purpose of this work is to develop a procedure employing a single model rendered over a smaller region but without the necessity of iterating the construction-prediction process. In order to accomplish this, the concept of performance-driven modeling is employed [41]. The main purpose of adopting it here is to narrow down the surrogate model domain so that it is oriented along the path corresponding to the maximum changes of the relevant antenna responses (here, reflection characteristics at and around the target operating frequencies), while maintaining its small size in the directions orthogonal to that path.

The directions of essential changes of antenna responses are identified by executing two optimization runs, one aiming at maximization of the fractional bandwidths of the antenna (symmetric with respect to the operating frequencies), the second aiming at minimizing the antenna reflection at the operating frequencies. Thus, two additional designs are obtained (apart from the nominal design $\boldsymbol{x}^{(0)}$ ) as

$$
\begin{gathered}
\boldsymbol{x}^{(1)}=\arg \min _{\boldsymbol{x}}\left\{-\min \left\{B_{1}(\boldsymbol{x}), \ldots, B_{N}(\boldsymbol{x})\right\}\right\} \\
\boldsymbol{x}^{(2)}=\arg \min _{\boldsymbol{x}}\left\{\max \left\{\left|S_{11}\left(\boldsymbol{x}, f_{01}\right)\right|, \ldots,\left|S_{11}\left(\boldsymbol{x}, f_{0 N}\right)\right|\right\}\right\}
\end{gathered}
$$

In (6), $B_{k}(\boldsymbol{x})$ is a symmetric part of the $k$ th bandwidth, i.e., $B_{k}(\boldsymbol{x})=2 \min \left\{f_{0 k}-f_{1 k}(\boldsymbol{x}), f_{2 k}(\boldsymbol{x})-f_{0 k}\right\}$ with $f_{1 k}$ and $f_{2 k}$ being the frequencies corresponding to $-10 \mathrm{~dB}$ level of $\left|S_{11}\right|$ (left- and right-hand-side ends of the $k$ th resonance). Note that both (6) and (7) are formulated in a minimax sense, i.e., the improvement of the worst case, the bandwidth in (6) and the reflection levels at the operating frequencies in (7). It should be reiterated that particular formulations of the problems (6) and (7) are motivated by the need for identifying the directions corresponding to possibly large change of the antenna responses at and around its operating frequencies. They do not need to coincide with the formulation of the original design problem used to generate the nominal design of the antenna of interest.

The problems (6), (7) are solved using trust-region gradient search [44] with the Jacobian matrix updated using the rank-one Broyden formula [45], [46]. The latter is sufficient because the expected design relocations $\left\|\boldsymbol{x}^{(0)}-\boldsymbol{x}^{(1)}\right\|$ and $\left\|\boldsymbol{x}^{(0)}-\boldsymbol{x}^{(2)}\right\|$ are limited. Consequently, the optimization process can be realized at a very low cost of around $1.5 n$ EM analyses, where $n$ is the parameter space dimensionality.

Let $s(t)=\left[s_{1}(t) \ldots s_{n}(t)\right]^{T}$ be a $t$-parameterized curve such that

$$
s_{j}(t)=a_{j 0}+a_{j 1} t+a_{j 2} t^{2}
$$

for $0 \leq t \leq 1$, so that $s(0)=x^{(1)}, s(0.5)=x^{(0)}$, and $s(1)=x^{(2)}$. The model coefficients can be found as

$$
\left[\begin{array}{lll}
a_{10} & \cdots & a_{n 0} \\
a_{11} & \cdots & a_{n 1} \\
a_{12} & \cdots & a_{n 2}
\end{array}\right]=\left[\begin{array}{ccc}
1 & 0 & 0 \\
1 & 0.5 & 0.25 \\
1 & 1 & 1
\end{array}\right]^{-1}\left[\begin{array}{l}
\left(\boldsymbol{x}^{(1)}\right)^{T} \\
\left(\boldsymbol{x}^{(0)}\right)^{T} \\
\left(\boldsymbol{x}^{(2)}\right)^{T}
\end{array}\right]
$$

Let $S(t)$ be the interval with the center at $\boldsymbol{s}(t)$ and the size $\boldsymbol{d}_{c}$ $=\left[\begin{array}{lll}d_{c 1} & \ldots & d_{c n}\end{array}\right]^{T}$, where $d_{c j}$ is a small multiplicity of the maximum design deviation $d_{\max }$, e.g., $2 d_{\max }$. We define the surrogate model domain $X_{S}$ as the set-theory union of the intervals $S(t)$ for $0 \leq t \leq 1$, i.e.,

$$
X_{S}=\bigcup_{0 \leq t \leq 1} S(t)
$$

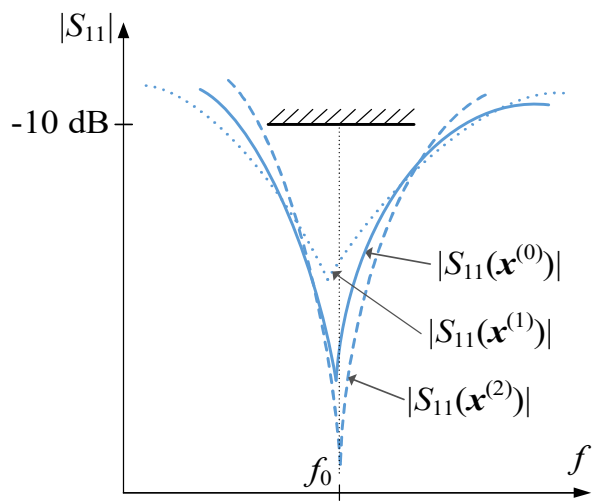

(a)

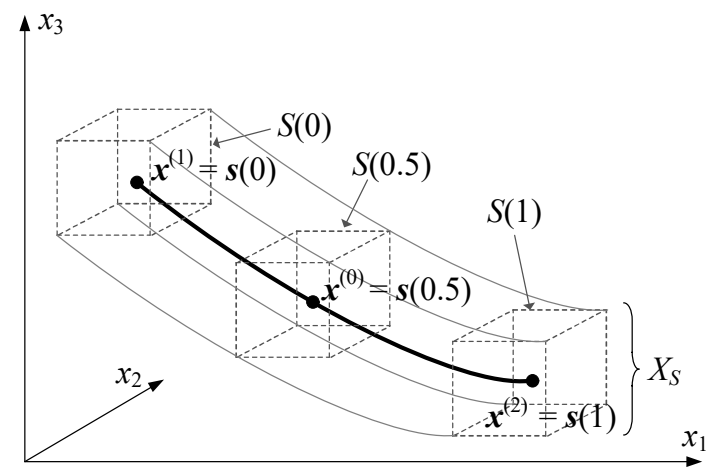

(b)

FIGURE 1. Yield optimization of narrow- and multi-band antennas using performance-driven surrogates: (a) reflection responses of an exemplary narrow-band antenna at the nominal design $x^{(0)}$, maximum bandwidth design $x^{(1)}$, and best matching (at $\left.f_{0}\right)$ design $x^{(2)}$. These designs determine the directions of the most significant response changes (from the point of view of the target operating bandwidth); (b) The reference designs $x^{(0)}$ through $x^{(2)}$ form a path (a parameterized curve $s(t)$ ). The union of intervals $S(t)$ (cf. (10) form the surrogate model domain $X s$. 
This domain contains the designs $\boldsymbol{x}^{(0)}, \boldsymbol{x}^{(1)}, \boldsymbol{x}^{(2)}$, and a vicinity of the entire curve $s(t)$ of the size $\boldsymbol{d}_{c}$. The set is of small size, yet it covers the directions of significant changes of the antenna responses, which are the most important from the point of view of manipulating the shape of the resonances, and, consequently, the performance figures such as yield. Due to a limited volume, a reliable surrogate model can be established in $X_{S}$ using a small number of training data samples. Furthermore, the entire optimization process can be performed within $X_{S}$ without the necessity of iterating the process, upon domain relocation, as in Algorithm 2 of Section II.B. Figure 1 provides a graphical illustration of the reference designs and the domain $X_{S}$.

Having defined the domain, the surrogate is constructed using kriging interpolation. The yield optimization is then carried out by directly solving the problem (3) at the level of surrogate, similarly as in Algorithm 1 of Section II.B. It should be emphasized that solving (3) using local algorithms (e.g., gradient-based procedures or pattern search methods) is sufficient, because the best-yield design is normally located in a relatively close vicinity of the nominal design. In particular, there is no need to use global search techniques such as population-based metaheuristics.

\section{DEMONSTRATION CASE STUDIES}

This section discusses numerical validation of the yield optimization procedure proposed in Section II.C. It is based on three antenna structures, a ring-slot antenna, a dual-band uniplanar dipole, and a triple-band dipole. Our methodology is compared to the surrogate-assisted algorithms of Section II.B. At the same time, the reliability of surrogatebased statistical analysis is validated using EM-driven Monte Carlo analysis run at the initial and optimized designs.

\section{A. CASE I: RING-SLOT ANTENNA}

The first verification example is a ring slot antenna shown in Fig. 2 [47], implemented on 0.76-mm-thick substrate of relative permittivity $\varepsilon_{r}=2.0$. The structure is excited through a microstrip line feeding a circular ground plane slot with defected ground structure (DGS). The latter is employed to suppress the antenna harmonic frequencies [48]. There are eight geometry parameters $\boldsymbol{x}=\left[\begin{array}{llllllll}l_{f} & l_{d} & w_{d} & r & s & s_{d} & o & g\end{array}\right]^{T}$. The computational model of the antenna is implemented in CST Microwave Studio ( 300,000 cells, simulation time $90 \mathrm{~s})$. The simulations of all the benchmark structures were performed on Intel Xeon 2.1 GHz dual-core CPU with 128 GB RAM.

The design objective is minimization of the in-band reflection for the frequency range $4.15 \mathrm{GHz}$ to $4.85 \mathrm{GHz}$ (center frequency $f_{0}=4.5 \mathrm{GHz}$ ). The nominal design is $\boldsymbol{x}^{(0)}=$ $\left[\begin{array}{llllllll}20.28 & 6.54 & 0.24 & 11.83 & 2.95 & 6.77 & 7.85 & 2.23\end{array}\right]^{T}$. The other reference designs $\boldsymbol{x}^{(1)}=\left[\begin{array}{llll}20.036 .30 & 0.2011 .842 .946 .747 .89\end{array}\right.$ $2.43]^{T}$, and $\boldsymbol{x}^{(2)}=\left[\begin{array}{lllllll}20.26 & 6.51 & 0.20 & 11.68 & 2.92 & 6.47 & 7.49\end{array}\right.$ $2.24]^{T}$, were obtained by solving (6) (maximum bandwidth design) and (7) (best reflection at $f_{0}=4.5 \mathrm{GHz}$ design). The cost of obtaining the reference designs is only 13 and 14 EM simulations, respectively.

Geometry parameter deviations are described by independent uniform probability distributions with the maximum deviation $d_{\max }=0.05 \mathrm{~mm}$. Table 1 shows the results of yield estimation at the initial design, yield optimization results, yield estimation at the final design for the proposed algorithm (Section II.C), as well as the reference algorithms (Section II.B). The size parameters $d_{c . k}$ defining the surrogate model domain were set to $2 d_{\max }$, and the model itself has been set up using 35 training samples (relative RMS error $0.5 \%$ ). The surrogate model for Algorithm 1 has been set using 400 samples within the domain of size $10 d_{\max }$ (relative RMS error $0.7 \%$ ). The surrogate models for Algorithm 2 have been set with 50 samples within the domain of size $3 d_{\max }$ (relative RMS error $0.4 \%$ at the first domain centered at $\left.\boldsymbol{x}^{(0)}\right)$. The design of experiments (sampling scheme) used in a construction of all surrogate models for this and the remaining verification examples is Latin Hypercube Sampling (LHS) [49]. The optimum design produced by the proposed approach is $\boldsymbol{x}^{*}=$ [20.18 6.43 0.2111 .852 .956 .787 .902 .31$]^{T}$. Figure 3 shows visualization of the Monte Carlo analysis at the nominal design and at $\boldsymbol{x}^{*}$. In each case, Monte Carlo analysis was performed using 500 samples generated according to the respective probability distribution.

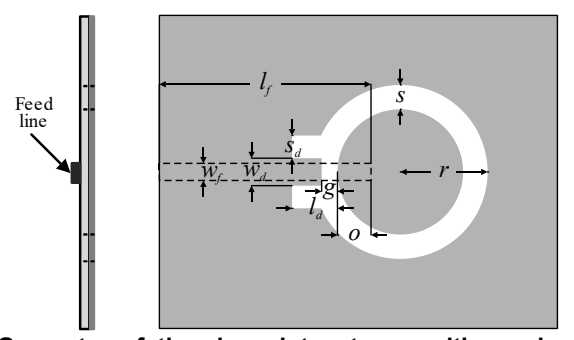

FIGURE 2. Geometry of the ring slot antenna with a microstrip feed (dashed line) [47]. The geometry parameters of the structure are $x=\left[l_{f} I_{d}\right.$ $\left.W_{d} r s S_{d} \circ g\right]^{T}$. Antenna is to be optimized for minimum in-band reflection within the frequency range $4.15 \mathrm{GHz}$ to $4.85 \mathrm{GHz}$ (center frequency $f_{0}=4.5$ $\mathrm{GHz}$ ). The nominal design is obtained by solving (4).

Table 1. Yield optimization of the ring slot antenna of Fig. 2

\begin{tabular}{cccccc}
\hline \hline \multirow{2}{*}{$\begin{array}{c}\text { Optimization } \\
\text { algorithm }\end{array}$} & $\begin{array}{c}\text { Estimated } \\
\text { by surrogate } \\
\text { model }\end{array}$ & $\begin{array}{c}\text { EM- } \\
\text { based }\end{array}$ & $\begin{array}{c}\text { Estimated } \\
\text { by surrogate } \\
\text { model }\end{array}$ & $\begin{array}{c}\text { EM- } \\
\text { based }\end{array}$ & $\begin{array}{c}\text { CPU } \\
\text { Cost }^{\$}\end{array}$ \\
\hline $\begin{array}{c}\text { Algorithm 1 } \\
\text { (Section II.B) }\end{array}$ & $81 \%$ & $81 \%$ & $92 \%$ & $93 \%$ & 400 \\
$\begin{array}{c}\text { Algorithm 2 } \\
\text { (Section II.B) }\end{array}$ & $81 \%$ & $81 \%$ & $91 \%$ & $91 \%$ & $150^{\#}$ \\
$\begin{array}{c}\text { This work } \\
\text { (Section II.C) }\end{array}$ & $81 \%$ & $81 \%$ & $91 \%$ & $91 \%$ & $62^{\&}$ \\
\hline \hline
\end{tabular}

${ }^{\$}$ Optimization cost in number of EM analyses of the antenna structure.

\# The algorithm convergence after three iterations (surrogate setup cost 50 training samples per iteration).

${ }^{\&}$ The cost includes training data acquisition (35 EM analyzes) and generation of the reference designs $\boldsymbol{x}^{(1)}$ and $\boldsymbol{x}^{(2)}$ (27 EM simulations in total). 


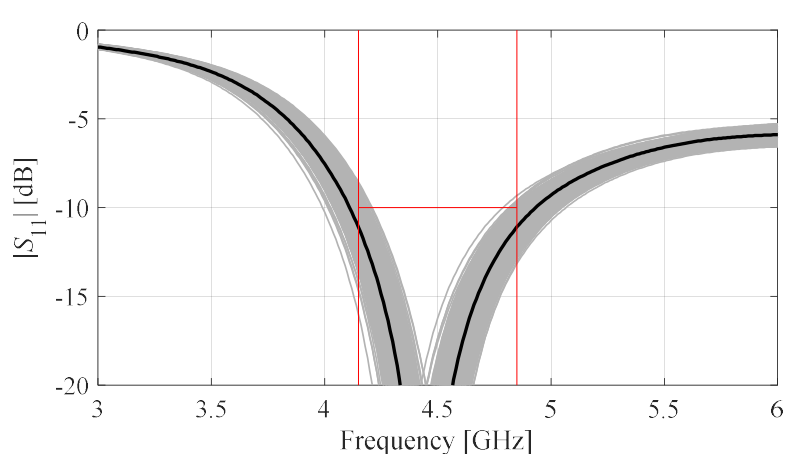

(a)

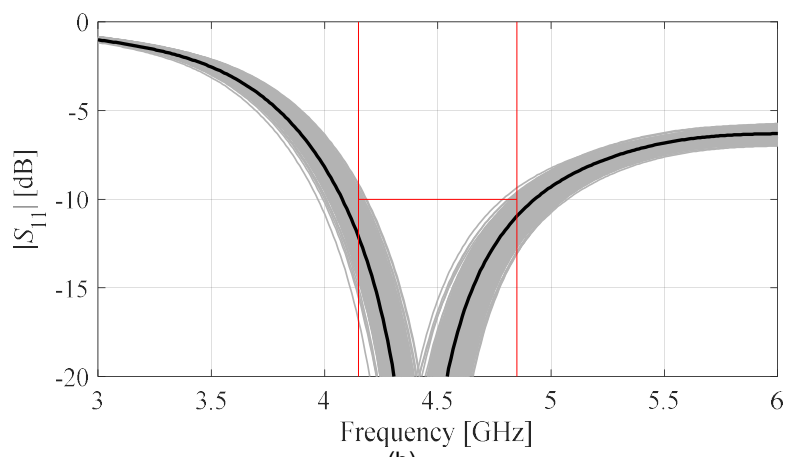

(b)

FIGURE 3. Monte Carlo analysis of antenna of Fig. 2 using EM simulations (gray plots): (a) nominal design, (b) yield-optimized design obtained using the algorithm proposed in this work. Black plots show the antenna response at the nominal and optimized designs, respectively.

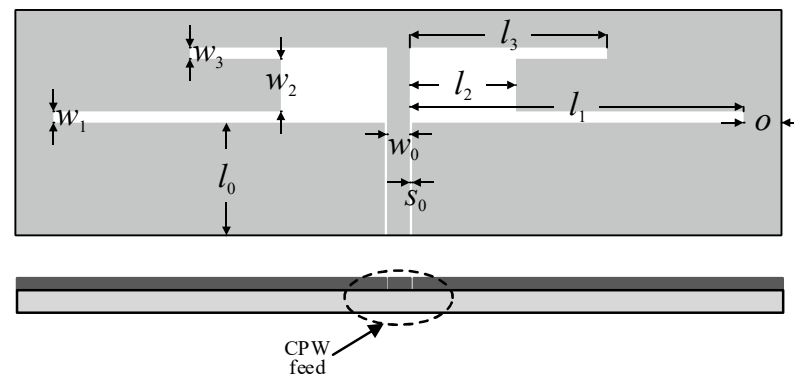

FIGURE 4. Geometry of a dual-band uniplanar dipole antenna [50]. The geometry parameters of the structure are $\left[\begin{array}{llllll}l_{1} & l_{2} & l_{3} & W_{1} & W_{2} & W_{3}\end{array}\right]^{T}$, fixed parameters: $I_{0}=30, w_{0}=3, s_{0}=0.15$ and $0=5$ (all dimensions in $\mathrm{mm}$ ). Antenna is to be optimized for minimum in-band reflection within 8percent symmetric bandwidths centered at the frequencies $f_{01}=3.0 \mathrm{GHz}$ and $f_{02}=5.5 \mathrm{GHz}$. The nominal design is obtained by solving (4).

The results of Table 1 indicate that appropriate constraining of the surrogate model domain as in the proposed approach allows for a significant reduction of the computational cost of the yield optimization process. On one hand, the cost of setting a reliable model itself is low due to the small volume of the domain. On the other hand, because the domain covers the relevant directions within the parameter space (i.e., those corresponding to the essential changes of the antenna response), there is no need to iterate the procedure: a one-shot approach is normally sufficient. This is corroborated by comparisons with Algorithms 1 and 2 of Section II.B. Both render the results of similar quality in terms of the final value of the yield, although the quality of the results produced by Algorithm 1 is slightly degraded due to a larger domain of the surrogate. The latter affects the model predictive power despite using a considerably larger number of training samples. Furthermore, EM-based Monte Carlo analysis executed for validation confirms reliability of yield estimation obtained from the surrogates.

\section{B. Case II: Dual-Band Uniplanar Dipole Antenna}

The second verification example is a dual-band uniplanar dipole antenna shown in Fig. 4 [50]. The antenna is implemented on a Rogers RO4350 substrate $\left(\varepsilon_{r}=3.5, h=\right.$ $0.76 \mathrm{~mm}$ ) and fed by a $50 \mathrm{Ohm}$ coplanar waveguide $(\mathrm{CPW})$. The adjustable variables are $\boldsymbol{x}=\left[\begin{array}{llllll}l_{1} & l_{2} & l_{3} & w_{1} & w_{2} & w_{3}\end{array}\right]^{T}$. Other parameters are fixed: $l_{0}=30, w_{0}=3, s_{0}=0.15$ and $o=5$ (all dimensions in $\mathrm{mm}$ ). The computational model of the antenna is implemented in CST Microwave Studio and evaluated using its time-domain solver $(\sim 100,000$ cells; simulation time 60 seconds).

The design objective is to minimize the in-band reflection within 8-percent symmetric bandwidths centered at the frequencies $f_{01}=3.0 \mathrm{GHz}$ and $f_{02}=5.5 \mathrm{GHz}$. The nominal design is $\boldsymbol{x}^{(0)}=\left[\begin{array}{llllll}30.47 & 11.6019 .20 & 0.472 .46 & 1.30\end{array}\right]^{T}$. The other two reference designs $\boldsymbol{x}^{(1)}=\left[\begin{array}{lllll}29.98 & 11.13 & 18.86 & 0.44 & 3.04\end{array}\right.$ $1.05]^{T}$ (maximum bandwidth) and $\boldsymbol{x}^{(2)}=\left[\begin{array}{ll}29.72 & 11.0319 .04\end{array}\right.$ $0.273 .010 .67]^{T}$ (best reflection at the operating frequencies) were obtained by solving (6) and (7), respectively. The reference designs are generated using only 11 and 10 EM simulations, respectively.

Similarly as in Section III.A, geometry parameter deviations are described by independent uniform probability distributions with the maximum deviation $d_{\max }=0.05 \mathrm{~mm}$. The results of yield optimization using the proposed algorithm and the benchmark methods have been gathered in Table 2.

The size parameters $d_{c . k}$ were set to $2 d_{\max }$ (training set size 53 samples, relative RMS error $0.8 \%$ ). The surrogate model for Algorithm 1 has been set using 800 samples within the domain of size $10 d_{\max }$ (relative RMS error $1.3 \%$ ). The surrogate models for Algorithm 2 have been set with 50 samples within the domain of size $3 d_{\max }$ (relative RMS error $0.9 \%$ at the first domain centered at $\left.\boldsymbol{x}^{(0)}\right)$. The optimum design produced by the proposed approach is $\boldsymbol{x}^{*}=[30.38$ $\left.\begin{array}{lllll}11.58 & 19.22 & 0.47 & 2.46 & 1.28\end{array}\right]^{T}$. Figure 5 shows visualization of the EM-based Monte Carlo analysis (using 500 random samples) at the nominal design and at $\boldsymbol{x}^{*}$.

The results obtained for this example are consistent with those discussed in Section III.A. The proposed approach outperforms both Algorithm 1 and Algorithm 2 of Section II.B in terms of the computational efficiency while providing the results of similar quality.

It should be noted that the predictive powers of the surrogate models are not as good as for the case of Section III.A. Therefore, slight discrepancies between the surrogatebased and EM-based yield estimations can be observed. The computational cost of yield optimization using the proposed method is reduced by about fifty percent compared to Algorithm 2, and it is significantly lower than for Algorithm 1. 


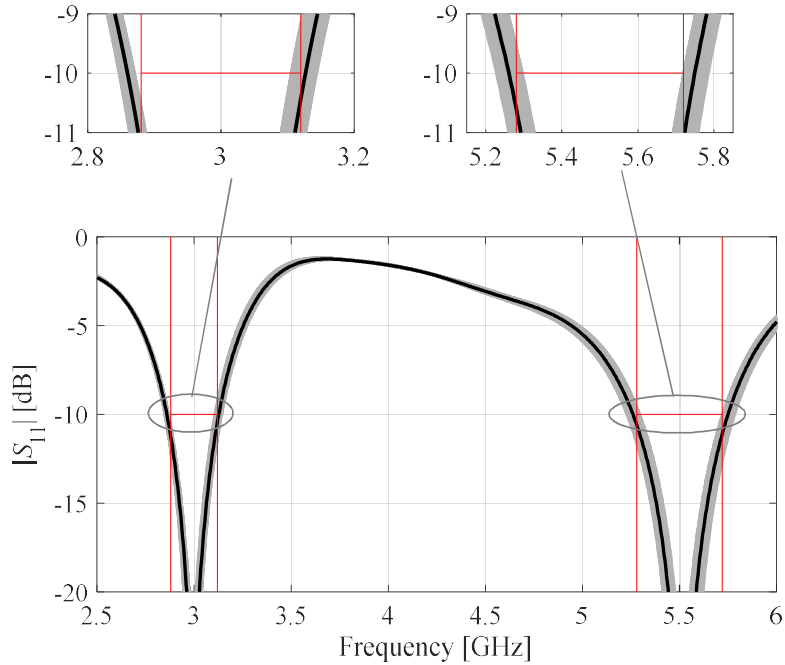

(a)

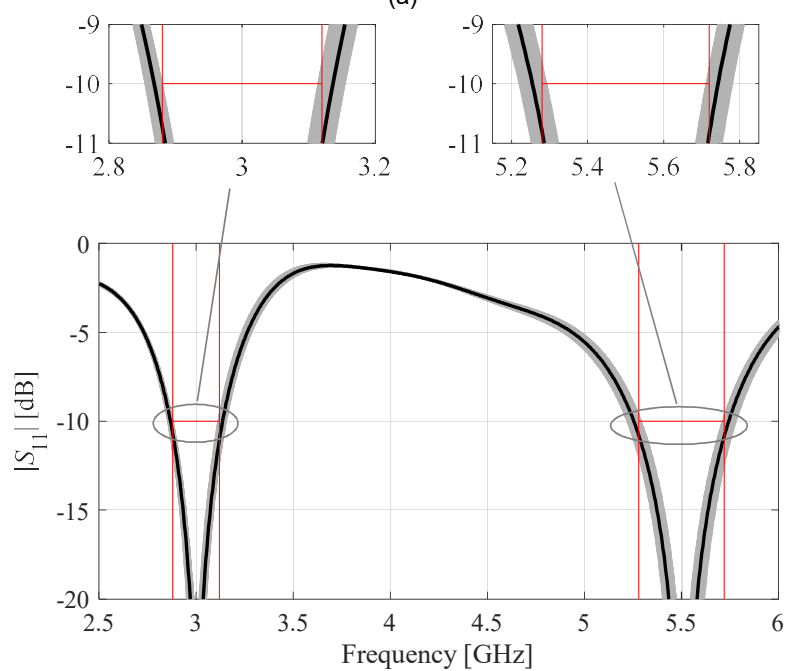

(b)

FIGURE 5. Monte Carlo analysis of antenna of Fig. 2 using EM simulations (gray plots): (a) nominal design, (b) yield-optimized design obtained using the algorithm proposed in this work. Black plots show the antenna response at the nominal and optimized designs, respectively.

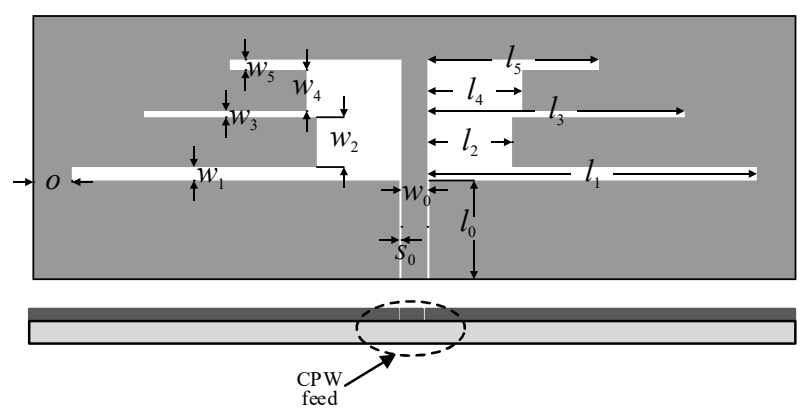

FIGURE 6. Geometry of a triple-band uniplanar dipole antenna [44]. The

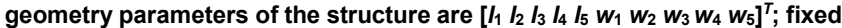
parameters: $l_{0}=30, w_{0}=3, s_{0}=0.15$ and $o=5$ (all dimensions in $\mathrm{mm}$ ). Antenna is to be optimized for minimum in-band reflection within 4percent symmetric bandwidths centered at the frequencies $f_{01}=2.45 \mathrm{GHz}$, $f_{02}=3.6 \mathrm{GHz}$, and $f_{03}=5.3 \mathrm{GHz}$. The nominal design is obtained by solving (4).

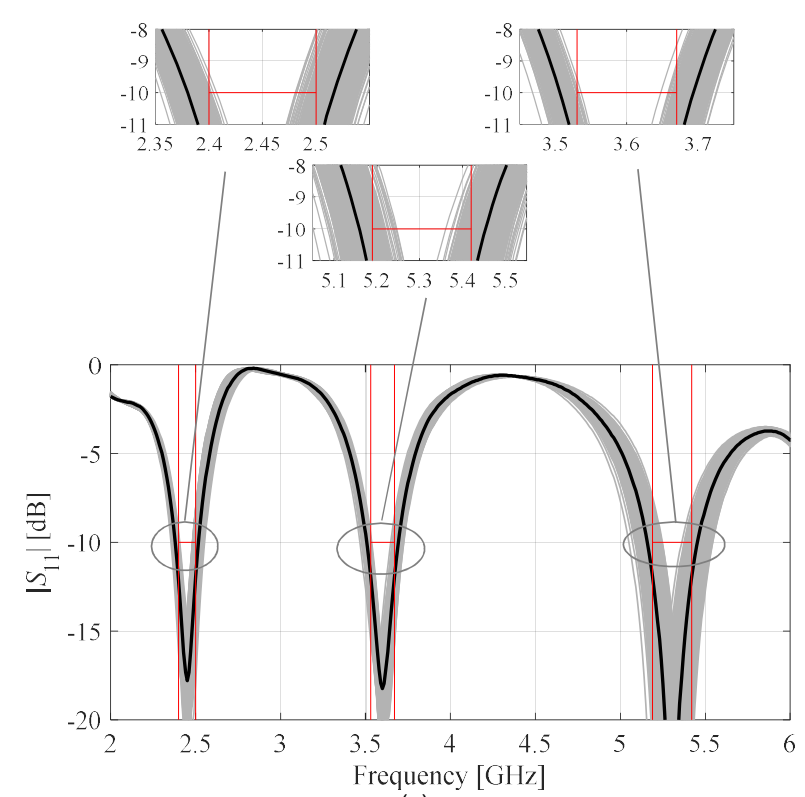

(a)

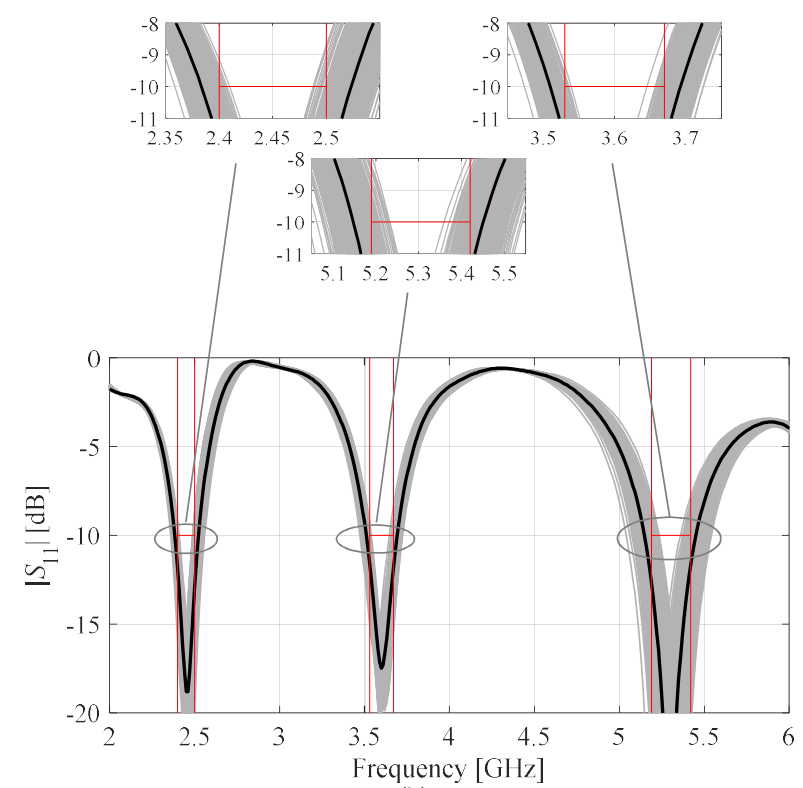

(b)

FIGURE 7. Monte Carlo analysis of antenna of Fig. 6 using EM simulations (gray plots): (a) nominal design, (b) yield-optimized design obtained using the algorithm proposed in this work. Black plots show the antenna response at the nominal and optimized designs, respectively.

\section{Case III: Triple-Band Uniplanar Dipole Antenna}

The last verification example is a triple-band uniplanar dipole antenna shown in Fig. 6. The structure is based on the design of [50] and implemented on RO4350 substrate. The

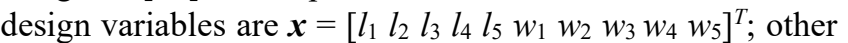
parameters are fixed: $l_{0}=30, w_{0}=3, s_{0}=0.15$ and $o=5$ (all dimensions in $\mathrm{mm}$ ). The EM-simulation model of the antenna is implemented in CST Microwave Studio ( 200,000 cells; simulation time 110 seconds).

The design objective is to minimize the in-band reflection within 4-percent symmetric bandwidths centered at the frequencies $f_{01}=2.45 \mathrm{GHz}, f_{02}=3.6 \mathrm{GHz}$, and $f_{03}=5.3 \mathrm{GHz}$. 
The nominal design is $\boldsymbol{x}^{(0)}=\left[\begin{array}{lll}35.42 & 11.5426 .078 .09 & 17.14\end{array}\right.$ $\left.\begin{array}{lllll}0.60 & 0.99 & 1.44 & 0.78 & 1.17\end{array}\right]^{T}$. The other two reference designs $\boldsymbol{x}^{(1)}=\left[\begin{array}{lllllllll}35.38 & 11.50 & 25.72 & 6.60 & 15.87 & 0.52 & 0.90 & 1.38 & 0.81\end{array}\right.$ $1.09]^{T}$ (maximum bandwidth) and $\boldsymbol{x}^{(2)}=\left[\begin{array}{lll}35.54 & 11.69 & 26.53\end{array}\right.$

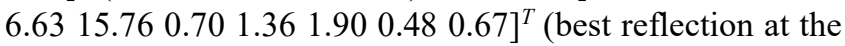
operating frequencies) were obtained by solving (6) and (7), respectively. The computational cost of obtaining $\boldsymbol{x}^{(1)}$ and $\boldsymbol{x}^{(2)}$ is $16 \mathrm{EM}$ antenna simulations in both cases.

Deviations of geometry parameters are described by the independent uniform probability distributions with the maximum deviation $d_{\max }=0.05 \mathrm{~mm}$. The results of yield optimization using the proposed algorithm and the benchmark methods have been gathered in Table 3. The surrogate model setup for all methods is the same as for the previous cases. The size parameters $d_{c . k}$ were set to $2 d_{\max }$ (training set size 100 samples, relative RMS error $1.3 \%$ ).

The surrogate model for Algorithm 1 has been set using 1,600 samples within the domain of size $10 d_{\max }$ (relative RMS error 2.8\%). The surrogate models for Algorithm 2 have been set with 100 samples within the domain of size $3 d_{\max }$ (relative RMS error $2.1 \%$ at the first domain centered at $\left.\boldsymbol{x}^{(0)}\right)$. The proposed algorithms rendered the yieldoptimized design $\boldsymbol{x}^{*}=\left[\begin{array}{l}35.3711 .5226 .08 .0917 .140 .591 .01 \\ 1.430 .00\end{array}\right.$ $\left.\begin{array}{lll}1.43 & 0.80 & 1.22\end{array}\right]^{T}$. Figure 7 shows visualization of the EMbased Monte Carlo analysis at the nominal design and at $\boldsymbol{x}^{*}$.

Table 2. Yield optimization of the dual-band antenna of Fig. 4

\begin{tabular}{cccccc}
\hline \hline \multirow{2}{*}{$\begin{array}{c}\text { Optimization } \\
\text { algorithm }\end{array}$} & $\begin{array}{c}\text { Initial Yield } \\
\text { Estimated } \\
\text { by surrogate } \\
\text { model }\end{array}$ & $\begin{array}{c}\text { EM- } \\
\text { based }\end{array}$ & $\begin{array}{c}\text { Estimated } \\
\text { by surrogate } \\
\text { model }\end{array}$ & $\begin{array}{c}\text { EM- } \\
\text { based }\end{array}$ & $\begin{array}{c}\text { CPU } \\
\text { Cost }^{\S}\end{array}$ \\
\hline $\begin{array}{c}\text { Algorithm 1 } \\
\text { (Section II.B) }\end{array}$ & $64 \%$ & $65 \%$ & $95 \%$ & $94 \%$ & 800 \\
$\begin{array}{c}\text { Algorithm 2 } \\
\text { (Section II.B) }\end{array}$ & $64 \%$ & $65 \%$ & $93 \%$ & $92 \%$ & $150^{\#}$ \\
$\begin{array}{c}\text { This work } \\
\text { (Section II.C) }\end{array}$ & $67 \%$ & $65 \%$ & $94 \%$ & $92 \%$ & $74^{\&}$ \\
\hline
\end{tabular}

${ }^{\$}$ Optimization cost in number of EM analyses of the antenna structure.

\# The algorithm convergence after three iterations (surrogate setup cost 50 training samples per iteration).

\& The cost includes training data acquisition (53 EM analyzes) and generation of the reference designs $\boldsymbol{x}^{(1)}$ and $\boldsymbol{x}^{(2)}$ (21 EM simulations in total).

Table 3. Yield optimization of the triple-band antenna of Fig. 6

\begin{tabular}{cccccc}
\hline \hline \multirow{2}{*}{$\begin{array}{c}\text { Optimization } \\
\text { algorithm }\end{array}$} & $\begin{array}{c}\text { Estimated } \\
\text { by surrogate } \\
\text { model }\end{array}$ & $\begin{array}{c}\text { EM- } \\
\text { based }\end{array}$ & $\begin{array}{c}\text { Estimated } \\
\text { by surrogate } \\
\text { model }\end{array}$ & $\begin{array}{c}\text { EM- } \\
\text { based }\end{array}$ & $\begin{array}{c}\text { CPU } \\
\text { Cost }^{\S}\end{array}$ \\
\hline $\begin{array}{c}\text { Algorithm 1 } \\
\text { (Section II.B) }\end{array}$ & $63 \%$ & $58 \%$ & $75 \%$ & $66 \%$ & 1,600 \\
$\begin{array}{c}\text { Algorithm 2 } \\
\text { (Section II.B) }\end{array}$ & $62 \%$ & $58 \%$ & $72 \%$ & $69 \%$ & $400^{\#}$ \\
$\begin{array}{c}\text { This work } \\
\text { (Section II.C) }\end{array}$ & $60 \%$ & $58 \%$ & $72 \%$ & $69 \%$ & $132^{\&}$ \\
\hline \hline
\end{tabular}

\$Optimization cost in number of EM analyses of the antenna structure.

\# The algorihm convergence after four iterations (surrogate setup cost 100 training samples per iteration).

${ }^{\&}$ The cost includes training data acquisition (100 EM analyzes) and generation of the reference designs $\boldsymbol{x}^{(1)}$ and $\boldsymbol{x}^{(2)}$ (32 EM simulations in total).
Also in this case, the proposed algorithm outperforms the benchmark and the overall results are consistent with those obtained for the previous cases. In this case, due to higher dimensionality of the parameter space and nonlinearity of the antenna response (three resonances), the numbers of training samples necessary to construct the surrogate models are noticeably larger.

Notwithstanding, Algorithm 2 (sequential approximate optimization) is not capable of finding as good design as those identified using Algorithm 1 and the proposed method. This is most likely due to restricted size of the domain of the local surrogate.

Furthermore, reliability of Algorithm 1 is not as good as for the previous examples because of limited predictive power of the surrogate $(2.8 \%)$. Finally, this last verification example pronounces even more the benefits of the approach proposed in this work, i.e., the capability of rendering highquality design at the low computational cost.

\section{CONCLUSION}

The paper proposed a novel yield optimization procedure for narrow- and multi-band antennas. Our methodology exploits the concept of performance-driven modeling to yield a fast and reliable surrogate model over a constrained domain spanned by the directions corresponding to the essential changes of the antenna characteristics. The appropriate selection of the reference designs for domain definition allows for rendering the model at a very low cost of a few dozens of EM analyses of the antenna at hand. At the same time the model covers the relevant regions of the parameter space so that the yield optimization process can be carried out in a oneshot manner (neither design relocation nor surrogate rebuilding is necessary). Numerical results obtained for the three test cases, a ring slot antenna, dual-band and triple band uniplanar dipoles, demonstrate the efficacy of the presented framework as well as its superiority over the benchmark (also surrogate assisted) procedures. Reliability of the procedure is confirmed by comparisons with EM-based Monte Carlo analysis at the nominal and the optimized designs. The future work will address generalization of the method for other types of antenna and microwave components.

\section{ACKNOWLEDGMENT}

The authors would like to thank Dassault Systemes, France, for making CST Microwave Studio available.

\section{REFERENCES}

[1] R. Lehmensiek and D.I.L. de Villiers, "Optimization of log-periodic dipole array antennas for wideband omnidirectional radiation," IEEE Trans. Ant. Prop., vol. 63, no. 8, pp. 3714-3718, 2015.

[2] A.A. Al-Azza, A.A. Al-Jodah, and F.J. Harackiewicz, "Spider monkey optimization: a novel technique for antenna optimization," IEEE Ant Wireless Prop. Lett., vol. 15, pp. 1016-1019, 2015.

[3] X. Jia and G. Lu, "A hybrid Taguchi binary particle swarm optimization for antenna designs," IEEE Ant. Wireless Prop. Lett., vol. 18, no. 8, pp. 1581-1585, 2019. 
[4] A. Pietrenko-Dabrowska and S. Koziel, „Computationally-efficient design optimization of antennas by accelerated gradient search with sensitivity and design change monitoring," IET Microwaves Ant. Prop., vol. 14, no. 2, pp. 165-170, 2020

[5] A. Narbudowicz, M. John, V. Sipal, X. Bao, and M.J. Amman, "Design method for wideband circularly polarized slot antennas," IEEE Trans. Ant. Prop., vol. 63, no. 10, pp. 4271-4279, 2015

[6] R. Biernacki, S. Chen, G. Estep, J. Rousset, J. Sifri, "Statistical analysis and yield optimization in practical RF and microwave systems," IEEE MTT-S Int. Microw. Symp. Dig., Montreal, pp. 1-3, 2012.

[7] A.S.O. Hassan, H.L. Abdel-Malek, A.S.A. Mohamed, T.M. Abuelfadl, and A.E. Elqenawy, "Statistical design centering of RF cavity linear accelerator via non-derivative trust region optimization," IEEE Int. Conf. Numerical EM Multiphysics Modeling Opt. (NEMO), pp. 1-3, 2015.

[8] S. Koziel, J. Bandler, A. Mohamed, and K. Madsen, "Enhanced surrogate models for statistical design exploiting space mapping technology," IEEE MTT-S Int. Microw. Symp. Dig., Long Beach, CA, pp. 1-4, 2005.

[9] I. Syrytsin, S. Zhang, G.F. Pedersen, K. Zhao, T. Bolin, and Z. Ying, "Statistical investigation of the user effects on mobile terminal antennas for 5G applications," IEEE Trans. Ant. Prop., vol. 65, no. 12, pp. 6596-6605, 2017.

[10] L. Leifsson, X. Du, and S. Koziel, "Efficient yield estimation of multiband patch antennas by polynomial chaos-based kriging," Int. J. Numer. Model., e2722, 2020.

[11] Z. Chen, Y. Xu, C. Wang, Z. Wen, Y. Wu, and R. Xu, "A large-signal statistical model and yield estimation of GaN HEMTs based on response surface methodology," IEEE Microwave Wireless Comp. Lett., vol. 26, no. 9, pp. 690-692, 2016

[12] L. Cai, Y. Zhao, W. Chen, P. Huang, X. Liu, and X. Zhang, "Selfheating aware EM reliability prediction of advanced CMOS technology by kinetic Monte Carlo method,” Int. Symp. Physical and Failure Anal. Int. Circuits, Hangzhou, China, July 2-5, 2019.

[13] C. Salis, N. Kantartzis, and T. Zygiridis, "Efficient stochastic EM studies via dimensionality reduction of polynomial chaos expansions," Int. Conf. Modern Circuits and Syst. Tech., Thessaloniki, Greece, May 7-9, 2018.

[14] M. Sengupta, S. Saxena, L. Daldoss, G. Kramer, S. Minehane, and J. Cheng, "Application-specific worst case corners using response surfaces and statistical models," IEEE Trans. Comput.-Aided Design Integr. Circuits Syst., vol. 24, no. 9, pp. 1372-1380, 2005.

[15] E. Sinkevich, "Worst-case models of RF front-end nonlinearity for discrete nonlinear analysis of electromagnetic compatibility," Int. Symp. Electr. Compatibility, Gothenburg, Sweden, Sept. 1-4, 2014.

[16] E. Matoglu, N. Pham, D. De Araujo, M. Cases, and M. Swaminathan, "Statistical signal integrity analysis and diagnosis methodology for high-speed systems," IEEE Trans. Adv. Packaging, vol. 27, no. 4, pp. 611-629, 2004

[17] J.E. Rayas-Sanchez, V. Gutierrez-Ayala, "EM-based statistical analysis and yield estimation using linear-input and neural-output space mapping," IEEE MTT-S Int. Microwave Symp. Digest (IMS), pp. $1597-1600,2006$

[18] J. Zhang, C. Zhang, F. Feng, W. Zhang, J. Ma, and Q.J. Zhang, "Polynomial chaos-based approach to yield-driven EM optimization," IEEE Trans. Microwave Theory Tech., vol. 66, no. 7, pp. 3186-3199, 2018.

[19] J. Du and C. Roblin, "Statistical modeling of disturbed antennas based on the polynomial chaos expansion," IEEE Ant. Wireless Prop. Lett., vol. 16, p. 1843-1847, 2017.

[20] M. Rossi, A. Dierck, H. Rogier, and D. Vande Ginste, "A stochastic framework for the variability analysis of textile antennas," IEEE Trans. Ant. Prop., vol. 62, no. 16, pp. 6510-6514, 2014.

[21] J.S. Ochoa and A.C. Cangellaris, "Random-space dimensionality reduction for expedient yield estimation of passive microwave structures," IEEE Trans. Microwave Theory Techn., vol. 61, no. 12, pp. 4313-4321, 2013

[22] H.L. Abdel-Malek, A.S.O. Hassan, E.A. Soliman, and S.A. Dakroury, "The ellipsoidal technique for design centering of microwave circuits exploiting space-mapping interpolating surrogates," IEEE Trans. Microwave Theory Techn., vol. 54, no. 10, pp. 3731-3738, 2006.
[23] M.C. Kennedy and A. O'Hagan, "Predicting the output from complex computer code when fast approximations are available", Biometrika vol. 87 , pp. 1-13, 2000

[24] D. Spina, F. Ferranti, G. Antonini, T. Dhaene, and L. Knockaert, "Efficient variability analysis of electromagnetic systems via polynomial chaos and model order reduction," IEEE Trans. Comp. Packaging Manufacturing Techn., vol. 4, no. 6, pp. 1038-1051, 2014

[25] B. Ma, G. Lei, C. Liu, J. Zhu, and Y. Guo, "Robust tolerance design optimization of a PM claw pole motor with soft magnetic composite cores," IEEE Trans. Magn., vol. 54, no. 3, paper No. 8102404, 2018.

[26] G. Scotti, P. Tommasino, and A. Trifiletti, "MMIC yield optimization by design centering and off-chip controllers," IET Proceedings Circuits, Devices and Systems, vol. 152, no. 1, pp. 54-60, Feb. 2005.

[27] A. Kouassi, N. Nguyen-Trong, T. Kaufmann, S. Lallechere, P. Bonnet, and C. Fumeaux, "Reliability-aware optimization of a wideband antenna," IEEE Trans. Ant. Prop., vol. 64, no. 2, pp. 450-460, 2016.

[28] Z. Ren, S. He, D. Zhang, Y. Zhang, and C.S. Koh, "A possibility-based robust optimal design algorithm in preliminary design state of electromagnetic devices," IEEE Trans. Magn., vol. 52, no. 3, paper No. 7001504, 2016

[29] J. Ko, J. Byun, J. Park, and H. Kim, "Robust design of dual band/polarization patch antenna using sensitivity analysis and Taguchi's method," IEEE Trans. Magn., vol. 47, no. 5, pp. 1258-1261, 2011

[30] A. Aubry, A. De Maio, Y. Huang, M. Piezzo, "Robust design of radar doppler filters." IEEE Trans. Signal Proc., vol. 64, no. 22, pp. 58485860,2016

[31] J.A. Easum, J. Nagar, P.L. Werner, and D.H. Werner, "Efficient multiobjective antenna optimization with tolerance analysis through the use of surrogate models," IEEE Trans. Ant. Prop., vol. 66, no. 12, pp. 6706-6715, 2018

[32] J. Zhang, F. Feng, W. Na, S. Yan, and Q. Zhang, "Parallel spacemapping based yield-driven EM optimization incorporating trust region algorithm and polynomial chaos expansion," IEEE Access, vol. 7, pp. 143673-143683, 2019

[33] Manfredi, D. Vande Ginste, D. De Zutter, and F.G. Canavero, "Improved polynomial chaos discretization schemes to integrate interconnects into design environments", IEEE Microw. Wireless Compon. Lett., vol. 23, no. 3, pp. 116-118, 2013

[34] M.R. Rufuie, E. Gad, M. Nakhla, and R. Achar, "Generalized Hermite polynomial chaos for variability analysis of macromodels embedded in nonlinear circuits", IEEE Trans. Compon. Packag. Manuf. Technol., vol. 4, no. 4, pp. 673-684, 2014

[35] S. Koziel and S. Ogurtsov, "Multi-level microwave design optimization with automated model fidelity adjustment," Int. J. RF and Microwave CAE, vol. 24, no. 3, pp. 281-288, 2013

[36] S. Koziel, "Fast simulation-driven antenna design using responsefeature surrogates," Int. J. RF \& Microwave CAE, vol. 25, no. 5, pp. 394-402, 2015.

[37] S. Koziel and J.W. Bandler, "Rapid yield estimation and optimization of microwave structures exploiting feature-based statistical analysis," IEEE Trans. Microwave Theory Tech., vol. 63, no. 1, pp. 107-114, 2015.

[38] S. Koziel and A. Pietrenko-Dabrowska, "Design-oriented computationally-efficient feature-based surrogate modelling of multiband antennas with nested kriging," AEU Int. J. Electronics Comm. vol. $120,2020$.

[39] S. Koziel and A. Pietrenko-Dabrowska, "Expedited feature-based quasi-global optimization of multi-band antennas with Jacobian variability tracking," IEEE Access, vol. 8, pp. 83907-83915, 2020.

[40] S. Koziel and A. Pietrenko-Dabrowska, "Performance-based nested surrogate modeling of antenna input characteristics," IEEE Trans. Ant Prop., vol. 67, no. 5, pp. 2904-2912, 2019.

[41] S. Koziel, "Low-cost data-driven surrogate modeling of antenna structures by constrained sampling," IEEE Antennas Wireless Prop. Lett., vol. 16, pp. 461-464, 2017.

[42] S. Koziel and A.T. Sigurdsson, "Triangulation-based constrained surrogate modeling of antennas," IEEE Trans. Ant. Prop., vol. 66, no. 8, pp. 4170-4179, 2018

[43] A. Pietrenko-Dabrowska and S. Koziel, "Antenna modeling using variable-fidelity EM simulations and constrained co-kriging," IEEE Access, vol. 8, no. 1, pp. 91048-91056, 2020. 
[44] A.R. Conn, N.I.M. Gould, and P.L. Toint, Trust Region Methods, MPS-SIAM Series on Optimization, 2000.

[45] C.G. Broyden, "A class of methods for solving nonlinear simultaneous equations," Math. Comp., vol. 19, pp. 577-593, 1965.

[46] S. Koziel and A. Pietrenko-Dabrowska, "Expedited optimization of antenna input characteristics with adaptive Broyden updates," Eng. Comp., vol. 37, no. 3, 2019.

[47] S. Koziel and A. Pietrenko-Dabrowska, Performance-Driven Surrogate Modeling of High-Frequency Structures, Springer, 2020.

[48] C.Y.D. Sim, M.H. Chang, and B.Y. Chen, "Microstrip-fed ring slot antenna design with wideband harmonic suppression," IEEE Trans. Ant. Prop., vol. 62, no. 9, pp. 4828-4832, 2014.

[49] B. Beachkofski and R. Grandhi, "Improved distributed hypercube sampling," American Institute of Aeronautics and Astronautics, paper AIAA 2002-1274, 2002.

[50] Y.-C. Chen, S.-Y. Chen, and P. Hsu, "Dual-band slot dipole antenna fed by a coplanar waveguide," IEEE Int. Symp. Ant. Prop., pp. 3589$3592,2006$.

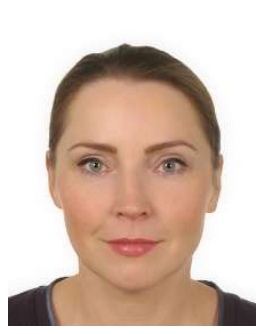

ANNA PIETRENKO-DABROWSKA received the M.Sc. and Ph.D. degrees in electronic engineering from Gdansk University of Technology, Poland, in 1998 and 2007, respectively. Currently, she is an Associate Professor with Gdansk University of Technology, Poland. Her research interests include simulationdriven design, design optimization, control theory, modeling of microwave and antenna structures, numerical analysis.

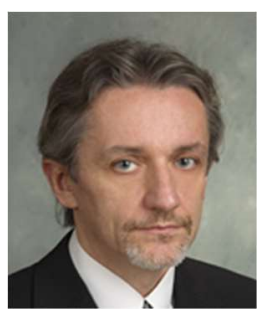

SLAWOMIR KOZIEL received the M.Sc. and $\mathrm{Ph} . \mathrm{D}$. degrees in electronic engineering from Gdansk University of Technology, Poland, in 1995 and 2000, respectively. He also received the M.Sc. degrees in theoretical physics and in mathematics, in 2000 and 2002, respectively, as well as the $\mathrm{PhD}$ in mathematics in 2003, from the University of Gdansk, Poland. He is currently a Professor with the Department of Engineering, Reykjavik University, Iceland. His research interests include $\mathrm{CAD}$ and modeling of microwave and antenna structures, simulation-driven design, surrogate-based optimization, space mapping, circuit theory, analog signal processing, evolutionary computation and numerical analysis.

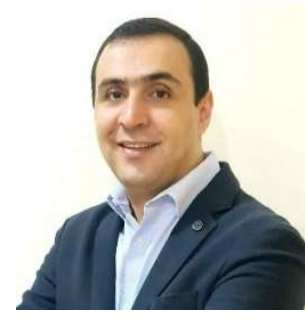

MU'ATH AL-HASAN received his B.A.Sc. degree in electrical engineering from the Jordan University of Science and Technology, Jordan, in 2005, the M.A.Sc in wireless communications from Yarmouk University, Jordan in 2008, and the Ph.D. degree in Telecommunication engineering from Institut National de la Recherche Scientifique (INRS), Université du Québec, Canada, 2015. From 2013 to 2014, he was with Planets Inc., California, USA. In May 2015, he joined Concordia University, Canada as postdoctoral fellowship. $\mathrm{He}$ is currently an Assistant Professor with Al Ain University, United Arab Emirates. His current research interests include antenna design at millimeter-wave and Terahertz, channel measurements in Multiple-Input and Multiple-Output (MIMO) systems, and Machine Learning and Artificial Intelligence in antenna design. 